\title{
Ontology mapping and merging aspects in semantic web
}

\begin{abstract}
Ontology has found application in multiple domains to represent relationships among classes and their instances. Ontology is being popularly applied as a tool for vocabulary conceptualization and optimization. To build vocabulary it requires mapping and merging of ontologies from heterogeneous sources of data. Many varied tools and techniques have been developed by researchers for ontology mapping and merging. This manuscript is an attempt to analyze and appreciate the tools available for ontology mapping and merging. The varied available tools automate integration, unification, aligning and merging of varied related ontologies. We also attempt to draw an efficient analysis among some popular tools while applying the PROMPT tool.
\end{abstract}

Keywords: mapping, merging, ontology, protégé, semantic web
Volume 4 Issue I - 20I I

\author{
Gagandeep Singh Narula,' Ritika Wason, ${ }^{2}$ \\ Vishal Jain, ${ }^{2}$ Anupam Baliyann ${ }^{2}$ \\ 'CSIR-NISTADS, India \\ 2Bharati Vidyapeeth's Institute of Computer Applications \& \\ Management (BVICAM), India
}

Correspondence: Gagandeep Singh Narula, CSIR-NISTADS, India,Tel 99998I3638, Email gagan.narula87@gmail.com

Received: October 29, 2017 | Published: January 24, 2018

\section{Introduction}

Let us assume there are two ontologies-each consisting of set of entities having classes, properties and instances and user wants to associate semantic relationships among entity components. According to Silva, ${ }^{1}$ the process of identifying semantic independences among entity components is termed as Ontology Mapping. Mapping has been applied variedly by several researchers in context of ontology related studies. ${ }^{2}$ Ontologies can be integrated, unified, merged and mapped to derive new set of relationships from existing ones. In Silva \& Howell, ${ }^{1,2}$ mapping is defined as set of formulae that consists of correlation coefficients to achieve semantic relationships among classes, properties and instances. In Tim, ${ }^{3}$ mapping is viewed as a process of maintaining correspondences among given ontologies and deriving similar concepts from them. The concepts may have different names but they point towards the same meaning. ${ }^{4-6}$ Varied techniques for ontology merging and alignment have also been suggested by several researchers. ${ }^{7-9}$ Merging of ontologies refers to generation of single independent logical ontology from given set of data sources while alignment of ontologies helps in achieving consistent ontologies. Valente, ${ }^{5}$ defines ontology mapping as a component that is responsible for providing mapping services so that concepts of ontology related to query can be mapped onto second ontology. This mapping service can be deployed in various applications that can be used for integration of ontologies. $^{7-9}$

Ontology Mapping is a key component in various applications as listed below:

\section{Integration of information using ontology}

Requires mapping of local ontologies into a single unified ontology. It applies the process of data integration to enable users to ask queries consistently without having access to data sources. Users ask queries through mediated ontology that involves use of agents to maintain interoperability and sharing between mediated ontology and local ontology. ${ }^{10,11}$

\section{Ontology merging}

Ontology merging and integration is defined as building new ontology from multiple ontologies consisting of overlapped subontologies $^{7}$ (Figure 1). The rest of the manuscript is structured as follows: Section II describes an overview of studies, techniques and theories conducted in context of ontology mapping. Section III briefly analyzes some significant ontology mapping tools. Section IV concludes the study.

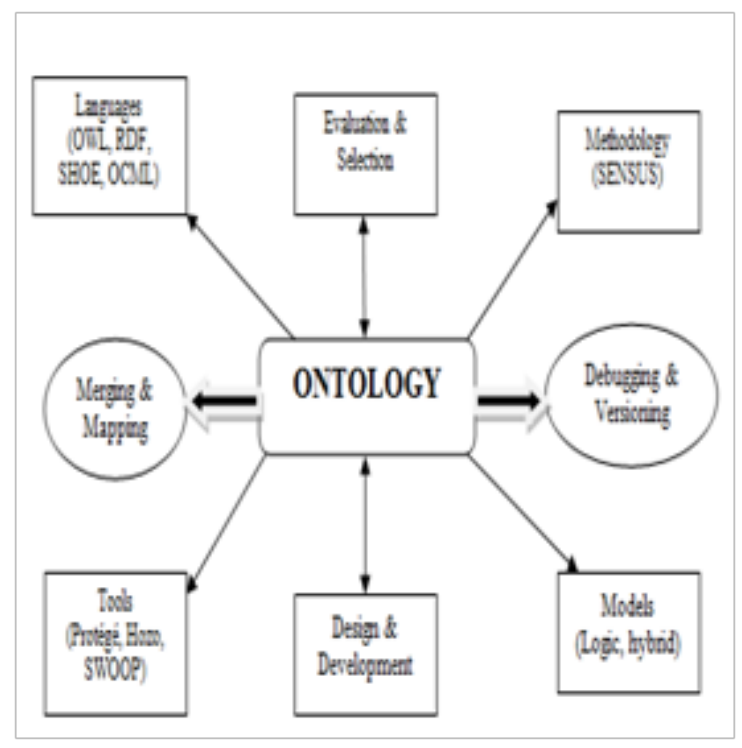

Figure I Ontology and its constituents. ${ }^{3}$

\section{Literature review}

In search engine examples have been used to illustrate the concepts from different ontologies in order to demonstrate relationship among them..$^{1,10-12}$ Visser et al..$^{13}$ introduced the concept of testing in identification of relations and constructs. It is followed by construction of concept lattice and mapping of lattice to distributed ontology. Lily ${ }^{13,14}$ is one of ontology mapping system that constructs semantic graph between concepts and relations on basis of linguistic features and structure of ontology. ASMOV ${ }^{14}$ is defined 
as an automated ontology mapping tool which computes similarity matching by analyzing textual and weighted features of different ontologies. Euzenat ${ }^{14}$ presented concept of virtual file technology to calculate similarity of concepts after analyzing linguistic features, terms and information stored in files. Various studies have been led by developers in $^{11,14-17}$ that describes matching criteria for integrating information from multiple data sources. Varied matching criteria have been applied in these works. Certain notable ones are:

a. Matching either by instance or schema information

b. Matching either by single elements or its structure

c. Matching on basis of language as well as constraints (keys and relationship among elements).

d. Matching on basis of auxiliary information (dictionaries).

Utilizing the above criteria the process of ontology mapping has been automated through different tools. The next section highlights few notable ones.

\section{Automatic ontology mapping tools}

Mapping tools are used to identify and define essential semantic correspondences automatically. ${ }^{15}$ Some notable ones are described in section 5.2 below:

\section{Requirements of an ideal ontology mapping approach}

Any ontology mapping approach must fulfill the following requirements:

a. It should be able to generate mappings automatically.

b. User should be able to accept, reject and add mappings.

c. Mappings should be defined and organized systematically.

d. Knowledge must be explored from derived mappings.

e. Hierarchical relationships between concepts and instances must be considered.

\section{Tools for ontology mapping and merging}

We describe succinct view of automatic ontology merging and mapping tools like Chimaera (web based), PROMPT (protégé plugin), FCA-Merge (ontologies on same domain), MOMIS (ontology clustering) and GLUE (machine learning). Further for clarity on working of these tools we also show results of an ontology mapping and merging simulation experiment on PROMPT using the protégé 3.4.1 plug-in.

Chimaera: This tool is used for merging and diagnosis of webbased distributed ontologies. Developed by Stanford University Knowledge System Laboratory (KSL) ${ }^{18}$ on the Ontolingua Distributed Collaborative Ontology Envoirnment.

Its salient features are as follows:

a. Mainly for light weight ontologies.

b. Combine semantically similar terms from multiple ontologies in order to produce unified ontologies.

c. Maintains relationship between disjoint terms. d. Produces two types of generation lists each having different purposes-Name resolution list and taxonomy resolution list. Former is used to merge terms from different ontologies and creates their taxonomy that is to be included in merged ontology while latter is used to maintain hierarchy of elements by applying heuristic techniques.

Prompt: It is an interactive tool for merging semi-automatic ontologies that serves as plug-in for Protégé [19]. Its salient features are as:

a. Besides merging ontology, it identifies locations for integration of ontologies, type of operations to be performed and resolves conflicts.

b. Interactive merging process i.e. several choices are being performed by user and PROMPT selects them automatically on basis of user preferences.

c. Components include names of classes and slots, instance hierarchy, facets and its values.

d. Handle conflicts like name conflicts, dangling references, redundant classes and slot value restrictions.

e. Provides iterative suggestions for merging concepts based on type of knowledge used.

To understand the process of ontology mapping and merging we simulated PROMPT on two bookshop ontologies. The figures below depict the results obtained. Protégé 3.4.1 was used for the experiment.

Further after mapping the above two ontologies on PROMPT they were merged as depicted. Figure 2-4 depict how efficiently ontologies can be mapped and merged through the PROMPT ontology merging tool. The experiment was conducted only on PROMPT as it was understood that the automated procedure is same for most of the tools which differ in their internal design and ontology mapping and merging criteria.

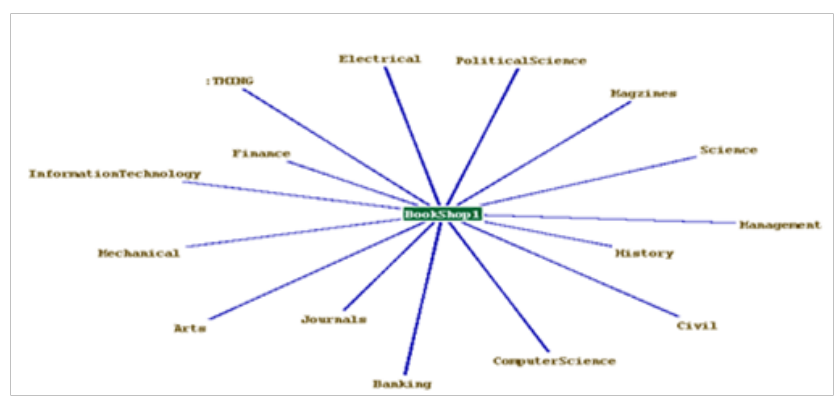

Figure 2 Source I Ontology Graph: BookShop I.

FCA-Merge: ${ }^{20}$ It deals with 3 step procedure for merging two ontologies.

a. Extraction of instances and evaluating formal contents $\mathrm{K} 1$ and $\mathrm{K} 2$.

b. Applying FCA merge algorithm for deriving common content and generation of concept lattice.

c. Produced result is merged into final ontology.

MOMIS: ${ }^{21}-$ It is an acronym for Mediator Environment for Multiple Information Sources that is developed by University of Modena. ${ }^{22}$ 
Its salient features are as follows:

a. Deals with data schema and global virtual schema in which latter one consists of mapping descriptions to perform semantic mapping between global and data schema.

b. Components include data model (ODM) and language (ODL) to describe sources of information.

c. Consists of wrapper that interacts with data schema. It translates data tags into representational language (ODL).

d. Generates thesaurus to create global virtual schema.

e. On basis of thesaurus contents, it describes relationship between inter and intra classes in clusters using hierarchical clustering techniques.

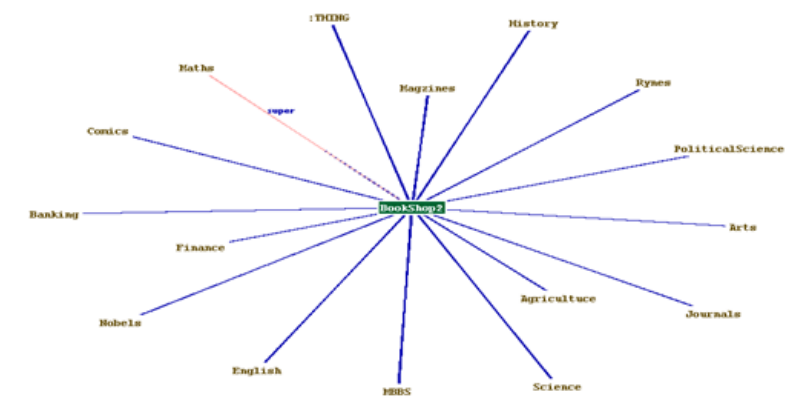

Figure 3 Source 2 Ontology Graph: BookShop2.

a. Distribution estimator-Firstly, it inputs two taxonomies T1 and T2 and their instances. Then it applies machine learning techniques to find pair of concepts between instances.

b. Similarity estimator: Above concepts acts as input to similarity estimator. Then it applies similarity function to compute similarity value for each pair of concepts. The output produced will be similarity matrix.

c. Relaxation Labeller: Similarity matrix along with constraints and knowledge acts as input. Then it searches for optional configuration mapping. The output produced will be mapping configuration.

\section{Conclusion}

Ontology is abbreviated as FESC (formal, explicit, shared and conceptualization of domain). ${ }^{24}$ Ontology displays information in hybrid manner such that it can be used by machines not only for displaying but also for automating, integrating and reusing given information across various existing applications. The paper explores terminologies related to ontology like mapping, integration, aligning, translation and merging. Ontology merging and integration is defined

Table I Comparative study of ontology mapping and merging systems

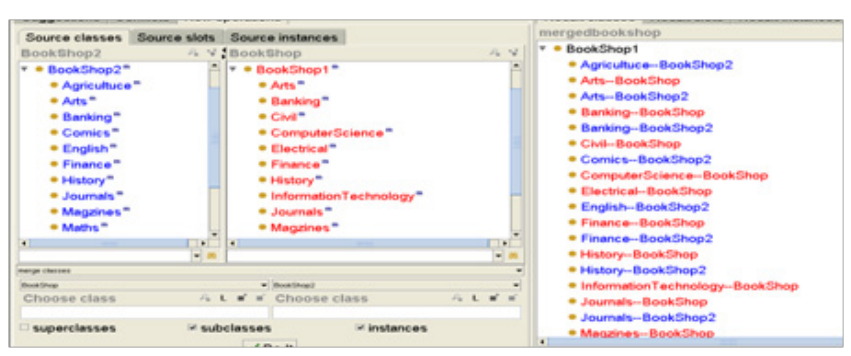

Figure 4 Ontology Merging: resultant merged Bookshop Ontology.

GLUE: ${ }^{23}$ It includes components- distribution estimator, similarity estimator and relaxation labeler (Figure 5).

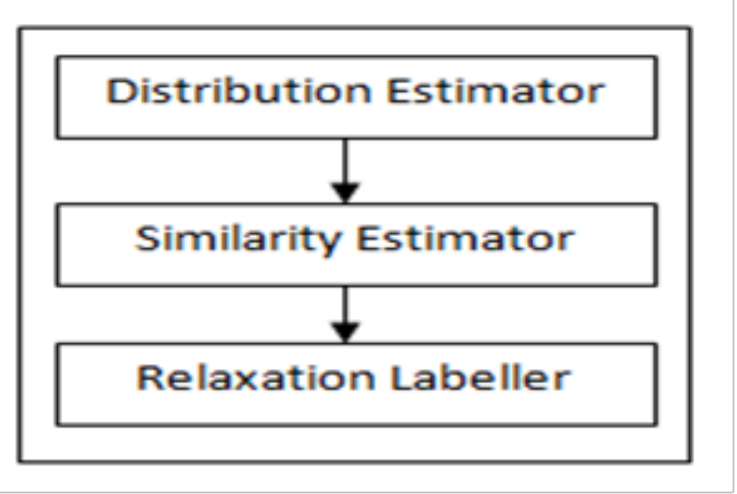

Figure 5 Components of GLUE.

as building new ontology from multiple ontologies consisting of overlapped sub ontologies. Ontology mapping is termed as relating similar (according to some metric) concepts or relations from different sources to each other by specifying the correspondence between them. Generally, process of mapping involves two tasks as stated below:

a. To discover independent semantic correspondences between ontology constituents (classes, properties and instances).

b. To define those discovered correspondences so that other ontologies can use them.

Ontology aligning makes source ontologies consistent and coherent by moving them together in cooperation. Ontology translation is defined as modifying the structure of ontology without changing its meaning while ontology transformation changes its structure as well as its meaning such that it is better than previous ontology with this manuscript we have attempted to give the reader an overview of the different ontology mapping and merging tools in a rather simplistic manner. Table 1 further highlights the differences among various available ontology mapping and merging systems.

\begin{tabular}{|c|c|c|c|c|c|c|c|c|c|}
\hline \multirow[b]{2}{*}{ S.no } & \multirow[b]{2}{*}{ Tool } & \multirow[b]{2}{*}{$\begin{array}{l}\text { Developed } \\
\text { by }\end{array}$} & \multirow[b]{2}{*}{$\begin{array}{l}\text { Ontology } \\
\text { representation }\end{array}$} & \multicolumn{4}{|c|}{ Mapping information } & \multirow[b]{2}{*}{ Mapping element } & \multirow[b]{2}{*}{$\begin{array}{l}\text { Mapping } \\
\text { cardinality }\end{array}$} \\
\hline & & & & $\begin{array}{l}\text { Define } \\
\text { concept }\end{array}$ & Graph & $\begin{array}{l}\text { Instances } \\
\text { structure }\end{array}$ & $\begin{array}{l}\text { User } \\
\text { input }\end{array}$ & & \\
\hline I & Chimaera & $\mathrm{KSL}^{18}$ & $\begin{array}{l}\text { OKBC } \\
\text { compliant }\end{array}$ & Yes & Yes & No & No & Concept, relations & I:01 \\
\hline 2 & PROMPT & $\begin{array}{l}\text { Stanford } \\
\text { Medical } \\
\text { Informatic }^{19}\end{array}$ & $\begin{array}{l}\text { OKBC } \\
\text { compliant }\end{array}$ & Yes & Yes & No & Yes & $\begin{array}{l}\text { Concept, relations and } \\
\text { constraint }\end{array}$ & I:n \\
\hline
\end{tabular}


Table Continued

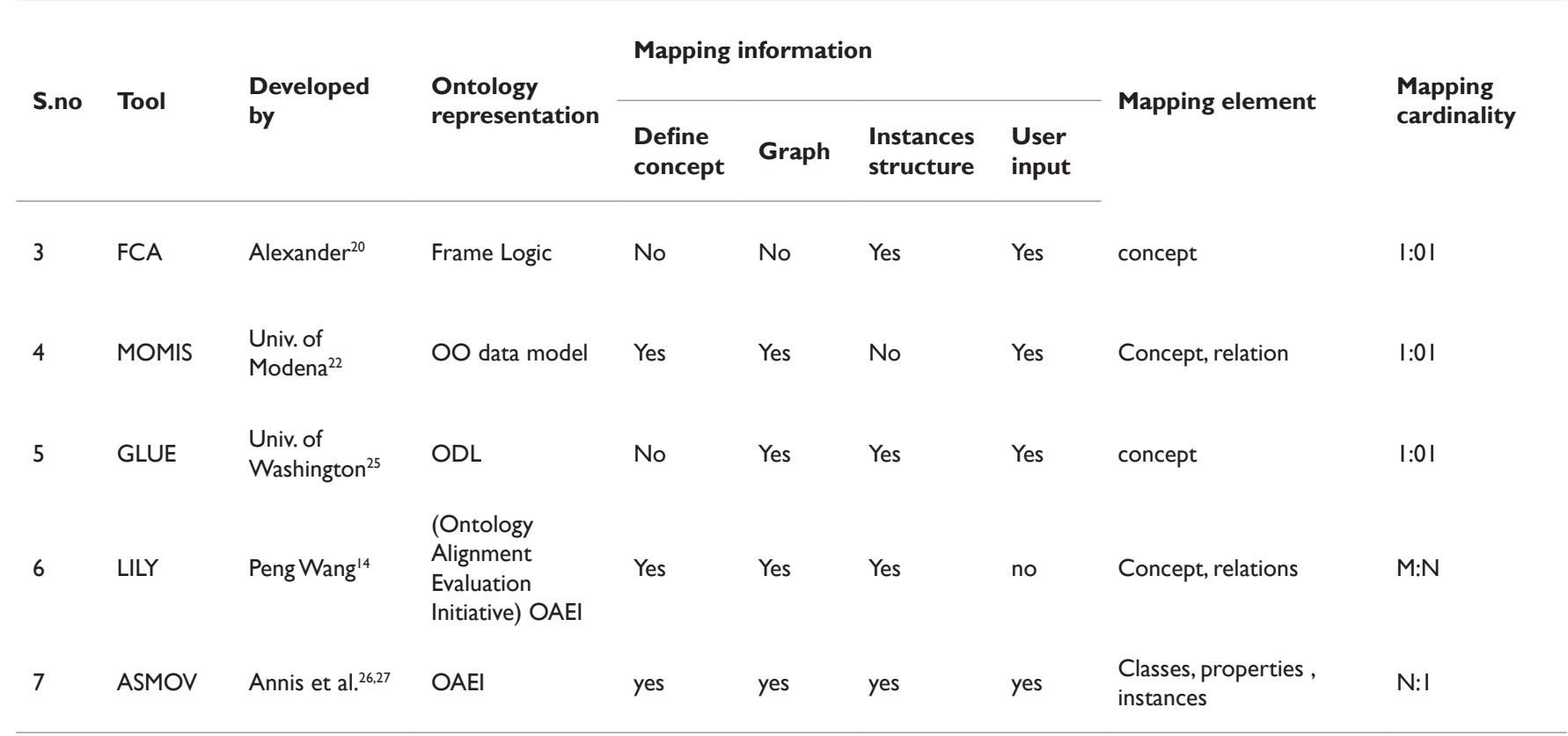

\section{Acknowledgments}

I Gagandeep Singh Narula would like to give my sincere thanks to Dr. Vishal Jain, Associate Professor at Bharati Vidyapeeth's Institute of Computer Applications and Management (BVICAM), New Delhi for guiding and motivating me towards research.

\section{Conflicts of interest}

None.

\section{References}

1. Silva N, Rocha J. Ontology mapping for interoperability in semantic web. Proc IADIS Int Conf. 2003;58:603-610.

2. Howell C. Machine Learning Methods of Mapping Semantic Web Ontologies. Wisc. 2008. p. 1-11.

3. Tim B, Lee B, Hendler J, Lassila O. Semantic Web will enable machines to comprehend semantic documents. 2001 p. 1-5.

4. Ehrig M, Sure Y. Ontology mapping - an integrated approach. Proc Eur Semant Web Symp. 2004. p. 76-91.

5. Valente A, Russ T, Mac Gregor R, et al. Building and (re)using an ontology of air campaign planning. IEEE Intell Syst. 1999;14(1):27-36.

6. Vishal Jain. Ontology : Development, Deployment and Merging Aspects in Semantic Web : An Overview. IMS Manthan. 2010;4(1):1-5:

7. Stumme G, Maedche A. Ontology Merging for Federated Ontologies on the Semantic Web. Proc Int Work Found Model Inf Integr. 2001. p. 413-418.

8. Yadav U, Singh Narula G, et al. Ontology Engineering and Development Aspects: A Survey. Int J Educ Manag Eng. 2016;6(3):9-19.

9. Chen L, Kokkinos I, Murphy K, et al. Semantic I Mage Segmentation with D Eep C on. 2014. p. 1-14.

10. X Su. A text categorization perspective for ontology mapping. Norw Dep Comput Inf. 2002. p. 1-9.
11. Olgu G, Elçi A. Integrating Ontologies by means of Semantic Partitioning. Canadian Semantic Web. 2006. p. 121-134.

12. Smart PR, Engelbrecht PC. An Analysis of the Origin of Ontology Mismatches on the Semantic Web. Knowledge Engineering: Practice and Patterns. 2008. p. 120-135.

13. Visser PRS, Beer MD, Bench-Capon TJM, et al. Resolving Ontological Heterogeneity in the KRAFT Project. Proc $10^{\text {th }}$ Int Conf Database Expert Syst Appl. 1999. p. 668-677.

14. Wang P, Xu B. Lily: Ontology alignment results for OAEI 2008. CEUR Workshop Proc. 2008;431(1):167-175.

15. Zhu Junwu. Survey on Ontology Mapping. Phys Procedia. 2012;24:18571862.

16. Cruz IF. Using a Layered Approach for Interoperability on the Semantic Web. Inf Syst IEEE. 2003.

17. Omelayenko B, Fensel D. A two-layered integration approach for product information in B2B e-commerce. Proc Second Int Conf Electron Commer Web Technol. 2001;2115:226-239.

18. McGuinness DL, Fikes R, Rice J, et al. The Chimaera ontology environment. Proc Natl Conf Artif Intell. 2000. p. 1123-1124.

19. Fridman N, Musen M. PROMPT: Algorithm and tool for automated ontology merging and alignment. Proc AAAI'00. 2000. p. 450-455.

20. G Stumme, Maedche A. FCA-MERGE:bottom-up merging of ontologies. Proceeding IJCAI'01 Proceedings of the $17^{\text {th }}$ international joint conference on Artificial intelligence. 2001;1:225-230.

21. De Bruijn J, Martın-Recuerda F, Manov D, et al. D4.2.1 State-of-the-art survey on Ontology merging and Aligning V1. EU-IST Integr Proj. 2004. p. 1-99.

22. Emilia ER. Ontology dynamics for Semantic Web: the MOMIS approach Dinamica delle ontologie per il Web Semantico: l'approccio di MOMIS. 2002.

23. Lourdusamy R, Ganapathy G. Feature analysis of ontology mediation tools. J Comput Sci. 2008;4(6):437-446. 
24. G Singh, Jain V. Information Retrieval (IR) through Semantic Web (SW): An Overview. 2014. p. 2-6.

25. Doan A, Madhavan J, Domingos P, et al. Ontology matching: A machine learning approach. Science. 2004;(80)13: 1-20.
26. Guedes A, Baião F, Revoredo K.) On the Identification and Representation of Ontology Correspondence Antipatterns. Proc $5^{\text {th }}$ Int Conf Ontol Semant Web Patterns (WOP'14). 2014;302:38-48.

27. Annis DS, Mosher DF, Roberts DD. NIH Public Access. 2009;27(4):339351 